\title{
HPC21
}

\section{Advanced IO Implementation \& Performances for Seismic Applications}

P.Y. Aquilanti (TOTAL E\&P R\&T USA LLC), M. Hugues* (TOTAL E\&P R\&T USA LLC), S. Jha (University of Houston), H. Calandra (TOTAL E\&P)

\section{Summary}

Oil \& Gas companies are facing an increasing challenge on extracting performances from parallel IO filesystems for check-pointing and writing results. Those efforts will increasingly become an issue due to the complexity introduced by the integration of more sophisticated seismic imaging equations in order to sustain the need of more detailed images to face the next exploration and production challenges. As current and future generations High Performance Computing (HPC) systems are evolving toward an increase computing power, IO bandwidth is remaining relatively constant. Henceforth, the need to manage IO efficiently at scale will become a strict requirement.

We will present an implementation and optimization study of ADIOS in the context of seismic imaging. We will exhibit a performance study made on a proxy application and on a RTM for several computing kernels for different HPC systems in the context of check-pointing. We will show that using ADIOS can provide good performances, manage more efficiently IO and reduce meta-data contention. We will highlight that advanced IO libraries such as ADIOS provide the opportunity to overcome the challenges of maintaining performances with high level IO interfaces at scale. 


\section{Advanced IO Implementations \& Performances for Seismic Applications}

Oil \& Gas companies are facing an increasing challenge on extracting performances from parallel IO filesystems for check-pointing and writing results. Those efforts will increasingly become an issue due to the complexity introduced by the integration of more sophisticated seismic imaging equations in order to sustain the need of more detailed images to face the next exploration and production challenges. As current and future generations High Performance Computing (HPC) systems are evolving toward an increase computing power, IO bandwidth is remaining relatively constant. Henceforth, the need to manage IO efficiently at scale will become a strict requirement.

As a matter for fact, efficiently managing parallel IO at scale can be very complex due to the various kinds of IO accesses that applications must manage: reading and writing big structured files, accessing small files, contiguous data and non-contiguous data. When considering other objectives such as code portability and stability, maintaining performance IO libraries can quickly become a daunting task. For this reason, advanced IO libraries such as the Adaptable IO System (ADIOS) library are considered as candidates to overcome those challenges. Indeed, the advantages of ADIOS is to provide a simple, yet powerful, interface to manage IO while preserving performances at scale.

We will present an implementation and optimization study of ADIOS in the context of seismic imaging. We will exhibit a performance study made on a proxy application and on a RTM for several computing kernels for different HPC systems in the context of check-pointing. We will show that using ADIOS can provide good performances, manage more efficiently IO and reduce meta-data contention. We will highlight that advanced IO libraries such as ADIOS provide the opportunity to overcome the challenges of maintaining performances with high level IO interfaces at scale. 